\title{
Local stability and parameter dependence of mild solutions for stochastic differential equations
}

Lijun Pan

"Correspondence: plj1977@126.com

School of Mathematics, Jia Ying University, Meizhou, Guangdong

514015, P.R. China

\begin{abstract}
For nonlinear stochastic equations $d x(t)=[A x(t)+f(t, x(t), \lambda)] d t+g(t, x(t), \lambda) d \omega(t)$ with parameter $\lambda$ in a Hilbert space, we show the existence and uniqueness of mild solutions. Provided that $f$ satisfies a locally Lipschitz condition and $g$ is a uniformly Lipschitz function, some sufficient conditions for $p(p \geq 2)$ moment locally exponential stability as well as almost surely exponential stability of mild solutions are obtained under a sufficiently small initial value $\xi$. Meanwhile, we also consider parameter dependence of stable mild solutions for the stochastic system if $f, g$ are sufficiently small Lipschitz perturbations in the parameter $\lambda$.
\end{abstract}

Keywords: mild solutions; exponential stability; parameter dependence

\section{Introduction}

Stochastic differential equation has attracted a great attention from both theoretical and applied disciplines since it has been successfully applied to problems in mechanics, economics, physics and several fields in engineering. For details, see [1-5] and the references therein. In recent years, existence, uniqueness, stability, invariant measures and other quantitative and qualitative properties of solutions to stochastic partial differential equations have been extensively considered by many authors. For example, in 1978, Haussmann [6] studied asymptotic stability of Itô equations in infinite dimensions. Caraballo [7] extended the results from Haussmann to stochastic partial differential equations with delay. In [8], Mao considered exponential stability in the mean square sense for the strong solutions of linear stochastic differential equations. Caraballo and Real [9] considered the stability for the strong solutions of semilinear stochastic evolution equations based on the ideas in [7]. Govindan [10,11] studied the existence and stability of mild solutions for stochastic partial differential equations by the comparison theorem. Caraballo and Liu [12] discussed the exponential stability for mild solutions of stochastic partial differential equations with delays by employing the well-known Gronwall inequality and stochastic analysis technique under the Lipschitz condition. Liu and Mao [13] established Razuminkhin-type exponential stability for mild solution of stochastic partial functional differential equations. Da Prato et al. [14] and Dawson [15] developed this topic by the semigroup approach [16]. In [17], Taniguchi et al. discussed the existence, uniqueness and asymptotic behavior of solutions to stochastic partial functional differential equations in a Hilbert space.

o2014 Pan; licensee Springer. This is an Open Access article distributed under the terms of the Creative Commons Attribution License (http://creativecommons.org/licenses/by/2.0), which permits unrestricted use, distribution, and reproduction in any medium, provided the original work is properly cited. 
Recently, Burton [18] has successfully extended the fixed point theorem to study the stability for deterministic systems. By employing the contraction mapping principle and stochastic analysis, Luo [19] has obtained some sufficient conditions which ensure exponential stability in $p(p \geq 2)$ moment and almost sure exponential stability for mild solutions of stochastic partial differential equations with delays. Following the ideas of Luo, Sakthivel and Luo investigated the existence and asymptotic stability in the $p$ th moment of mild solutions of nonlinear impulsive stochastic differential equations with infinite delay in [20].

Our main objective is to obtain local stability of mild solutions for stochastic parameter systems, with two novelties when compared with the former work in this area:

1. We consider $p(p \geq 2)$ th moment locally exponential stability as well as almost surely exponential stability of mild solutions under sufficiently small nonlinear perturbations and sufficiently small initial value.

2. We first discuss parameter dependence of stable mild solutions under sufficiently small Lipschitz perturbation in the parameter space $Y$.

The rest of this paper is organized as follows. In Section 2, stochastic differential equations with parameter are presented together with some definitions of mild solutions. In Section 3, existence, uniqueness, stability and parameter dependence of mild solutions are derived. An example is given to illustrate our results in Section 4. At last, we present some conclusions of our paper in Section 5.

\section{Preliminaries}

Let $H$ be a real separable Hilbert space with the inner product $(\cdot, \cdot)$ and the norm $\|\cdot\|$, and let $K$ be another real separable Hilbert space with the inner product $(\cdot, \cdot)_{K}$ and the norm $\|\cdot\|_{K} \cdot L(K, H)$ denotes the space of bounded operators from $K$ to $H$. Let $(\Omega, \mathscr{F}, P)$ be a complete probability space equipped with a complete family of right-continuous increasing sub $\sigma$-algebras $\left\{\mathscr{F}_{t}, t \geq 0\right\}$ satisfying $\mathscr{F}_{t} \subset \mathscr{F}$.

Let $\beta_{n}(t), n=1,2, \ldots$, be a sequence of real-valued one-dimensional standard Brownian motions mutually independent over $(\Omega, \mathscr{F}, P)$. Set

$$
w(t)=\sum_{n=1}^{\infty} \sqrt{\lambda_{n}} \beta_{n}(t) \xi_{n}, \quad t \geq 0,
$$

where $\lambda_{n} \geq 0(n=1,2, \ldots)$ are nonnegative real numbers and $\left\{\xi_{n}\right\}(n=1,2, \ldots)$ is a complete orthonormal basis in $K$. Let $Q \in L(K, K)$ be an operator defined by $Q \xi_{n}=\lambda_{n} \xi_{n}$ with a finite trace $\operatorname{Tr}(Q)=\sum_{n=1}^{\infty} \lambda_{n}<\infty$. Then the above $K$-valued stochastic process $\omega(t)$ is called a $Q$ Wiener process.

Let $\varphi \in L(K, H)$ and define

$$
\|\varphi\|_{L_{2}^{0}}^{2}=\operatorname{Tr}\left(\varphi Q \varphi^{*}\right)=\left\{\sum_{n=1}^{\infty}\left\|\sqrt{\lambda_{n}} \varphi \xi_{n}\right\|^{2}\right\} .
$$

If $\|\varphi\|_{L_{2}^{0}}<\infty$, then $\varphi$ is called a $Q$-Hilbert-Schmidt operator, where $L_{2}^{0}(K, H)$ denotes the space of all Q-Hilbert-Schmidt operators $\varphi: K \rightarrow H$.

We denote by $L^{p}(\Omega, H)(p \geq 2)$ the collection of all strongly-measurable, $p$-integrable $H$-valued variables with the norm $\|x(\cdot)\|_{L^{p}}=\left(E\|x(\cdot ; \omega)\|_{H}^{p}\right)^{\frac{1}{p}}$, where $E$ is defined by $E(h)=$ $\int_{\Omega} h(\omega) d P$. 
We consider the following stochastic differential equations with parameter in a Hilbert space:

$$
\begin{aligned}
& d x(t)=[A x(t)+f(t, x(t), \lambda)] d t+g(t, x(t), \lambda) d \omega(t), \quad t \geq 0, \\
& x(0)=\xi,
\end{aligned}
$$

where $\xi$ is an $H$-valued random variable, $A$ is the infinitesimal generator of a semigroup of bounded linear operators $S(t), t \geq 0, f: R^{+} \times H \times Y \rightarrow H, g: R^{+} \times H \times Y \rightarrow L(K, H)$ are all Borel measurable, $Y$ is a Banach space (which is the parameter space).

Now, we recall the mild solutions for Eq. (1) as follows.

Definition 2.1 A stochastic process $\{x(t, \xi, \lambda): t \in[0, T], \lambda \in Y\}, 0 \leq T<\infty$, is called a mild solution of Eq. (1) if

(a) $x(t, \xi, \lambda)$ is adapted to $\mathscr{F}_{t}, t \geq 0$;

(b) $x(t, \xi, \lambda) \in H$ has a càdlàg path on $t \in[0, T]$ almost surely;

(c) for arbitrary $t \in[0, T]$,

$$
x(t, \xi, \lambda)=S(t) \xi+\int_{0}^{t} S(t-s) f(s, x(s), \lambda) d s+\int_{0}^{t} S(t-s) g(s, x(s), \lambda) d \omega(s) .
$$

We assume that the following conditions hold:

(i) $A$ is the infinitesimal generator of a semigroup of bounded linear operators $S(t)$, $t \geq 0$, and there exist $M \geq 1, \eta>0$ such that $\|S(t)\|_{H} \leq M e^{-\eta t}$ for $t \geq 0$;

(ii) there exist constants $c>0, q>0$ such that for $t \geq 0, x, y \in H, \lambda, \mu \in Y$,

$$
\|f(t, x, \lambda)-f(t, y, \lambda)\|_{H} \leq c\|x-y\|_{H}\left(\|x\|_{H}^{q}+\|y\|_{H}^{q}\right)
$$

and

$$
\|f(t, x, \lambda)-f(t, x, \lambda)\|_{H} \leq c\|\lambda-\mu\|_{H}\|x\|_{H}^{q+1}
$$

(iii) there exists $d>0$ such that for $t \geq 0, x, y \in H, \lambda, \mu \in Y$,

$$
\|g(t, x, \lambda)-g(t, y, \lambda)\|_{L_{2}^{0}} \leq d\|x-y\|_{H}
$$

and

$$
\|g(t, x, \lambda)-g(t, x, \mu)\|_{L_{2}^{0}} \leq d\|\lambda-\mu\|_{Y}\|x\|_{H}
$$

(iv) $q+1 \leq p$;

(v) $2^{p} M^{p} d^{p} C_{p}\left(\frac{1}{2 \eta}\right)^{\frac{p}{2}}<1$, where $C_{p}=(p(2 p-1))^{p}$.

For example, if

$$
f(t, x, \lambda)=\sum_{j=1}^{m} \sum_{i=1}^{n} \lambda^{j} f_{i}(t, x),
$$


and

$$
g(t, x, \lambda)=\sum_{j=1}^{m} \sum_{i=1}^{n} \lambda^{j} g_{i}(t, x)
$$

for some functions $f_{i}, g_{i}: R^{+} \times H \rightarrow H$, and there exist constants $c_{i}>0, d_{i}>0, i=1,2, \ldots, n$, $q>0$ such that for $t \geq 0, x, y \in H$,

$$
\left\|f_{i}(t, x)-f_{i}(t, y)\right\|_{H} \leq c_{i}\|x-y\|_{H}\left(\|x\|_{H}^{q}+\|y\|_{H}^{q}\right),
$$

and

$$
\left\|g_{i}(t, x)-g_{i}(t, y)\right\|_{L_{2}^{0}} \leq d_{i}\|x-y\|_{H}
$$

for each $t \geq 0, x, y \in H$, then conditions (ii) and (iii) hold for $\lambda, \mu$ in a small neighborhood of zero.

Set $B(\delta)=\left\{x \in H: E\|x\|_{H}^{p} \leq \delta\right\}, \delta>0$. Then we have the following definitions of locally exponential behavior for mild solutions.

Definition 2.2 Let $p \geq 2$ be an integer, mild solution $x(t, \xi, \lambda)$ of Eq. (1) is said to be $p$ th moment locally exponentially stable if there exist $\gamma>0, L \geq 1$ such that

$$
E\|x(t, \xi, \lambda)\|_{H} \leq L e^{-\gamma t} E\|\xi\|_{H}^{p}
$$

for any initial value $\xi \in B(\delta), \lambda \in Y$ and $t \geq 0$.

Definition 2.3 The mild solution $x(t, \xi, \lambda)$ of Eq. (1) is said to be almost surely exponentially stable if for any initial value $\xi \in B(\delta)$ and for any $\lambda \in Y$, there exists $v>0$ such that

$$
\limsup _{t \rightarrow+\infty} \frac{1}{t} \log \|x(t, \xi, \lambda)\|_{H} \leq-v, \quad \text { a.s. }
$$

Lemma 2.1 [2] For any $r \geq 1$ and for arbitrary $L_{2}^{0}(K, H)$-valued predictable process $\Phi(\cdot)$,

$$
\sup _{s \in[0, t]} E\left\|\int_{0}^{s} \Phi(u) d \omega(u)\right\|^{2 r} \leq C_{r}\left(\int_{0}^{t}\left(E\|\Phi(s)\|_{L_{2}^{0}}^{2 r}\right)^{\frac{1}{r}} d s\right)^{r}, \quad t \geq 0,
$$

where $C_{r}=(r(2 r-1))^{r}$.

\section{Stability and parameter dependence}

In this section, we present and prove our main results. We consider the set $Z(\delta)=\{(t, \xi)$ : $0 \leq t \leq T, \xi \in B(\delta)\}$.

Theorem 3.1 Assume that (i)-(v) hold. Then, for any $\delta>0$ sufficiently small and for any $\lambda \in Y$, given $(t, \xi) \in Z(\delta)$, there exists a unique stochastic process $x(t, \xi, \lambda)$ satisfying (2). Moreover, the following properties hold: 
1. There exists $0<\alpha<p \eta$ such that

$$
2^{p} M^{p} d^{p} C_{p}\left[\frac{p}{2(p \eta-\alpha)}\right]^{\frac{p}{2}}<1
$$

2. $x(t, \xi, \lambda)$ is pth moment locally exponentially stable, that is, there exists $L \geq 1$ such that

$$
E\|x(t, \xi, \lambda)\|_{H}^{p} \leq L e^{-\alpha t} E\|\xi\|_{H}^{p}
$$

for any $\lambda \in Y$ and $(t, \xi) \in Z(\delta)$;

3. There exists $K>1$ such that

$$
E\|x(t, \xi, \lambda)-x(t, \xi, \mu)\|_{H}^{p} \leq K\|\lambda-\mu\|_{Y}^{p} e^{-\alpha t}
$$

for any $\lambda, \mu \in Y$ and $(t, \xi) \in Z(\delta)$.

Proof By (iv), it follows that there exists $0<\alpha<\eta$ such that (3) holds. We consider the space $\chi$ of all $\mathscr{F}$-adapted processes $x=x(t, \xi, \lambda):[0,+\infty) \times B(\delta) \times Y \rightarrow H$ such that

1. $x(0, \xi, \lambda)=\xi$ for any $\lambda \in Y$;

2. the norm $\|x\|_{\chi}=\frac{1}{2 M} \sup _{t \geq 0}\left[e^{\alpha t} E\|x(t, \xi, \lambda)\|_{H}^{p}\right]$ satisfies $\|x\|_{\chi} \leq \delta$.

We can easily see that $\chi$ is a complete metric space. Define an operator $J_{\lambda}: \chi \rightarrow \chi$ by

$$
\begin{aligned}
J_{\lambda}(x)(t, \xi)= & S(t) \xi(0)+\int_{0}^{t} S(t-s) f(s, x(s, \xi, \lambda), \lambda) d s \\
& +\int_{0}^{t} S(t-s) g(s, x(s, \xi, \lambda), \lambda) d \omega(s) \\
= & \sum_{i=1}^{3} I_{i}(t)
\end{aligned}
$$

for any $x \in \chi$. We first verify the continuity of $J_{\lambda}$ in $\chi$. Let $x \in \chi, t_{1} \geq 0$, and $|\epsilon|$ be sufficiently small, then

$$
E\left\|J_{\lambda}(x)\left(t_{1}+\epsilon\right)-J_{\lambda}(x)\left(t_{1}\right)\right\|_{H}^{p} \leq 3^{p-1} \sum_{i=1}^{3} E\left\|I_{i}\left(t_{1}+\epsilon\right)-I_{i}\left(t_{1}\right)\right\|_{H}^{p} .
$$

Obviously,

$$
E\left\|I_{i}\left(t_{1}+\epsilon\right)-I_{i}\left(t_{1}\right)\right\|_{H}^{p} \rightarrow 0, \quad i=1,2
$$

as $\epsilon \rightarrow 0$. By Lemma 2.1, we have

$$
\begin{aligned}
& E\left\|I_{3}\left(t_{1}+\epsilon\right)-I_{3}\left(t_{1}\right)\right\|_{H}^{p} \\
& \leq 2^{p-1} E\left\|\int_{0}^{t_{1}}\left(S\left(t_{1}+\epsilon-s\right)-S\left(t_{1}-s\right)\right) g(s, x(s, \xi, \lambda), \lambda) d \omega(s)\right\|_{H}^{p} \\
& \quad+2^{p-1} E\left\|\int_{t_{1}}^{t_{1}+\epsilon} S\left(t_{1}+\epsilon-s\right) g(s, x(s, \xi, \lambda), \lambda) d \omega(s)\right\|_{H}^{p}
\end{aligned}
$$




$$
\begin{aligned}
\leq & 2^{p-1} C_{p}\left(\int_{0}^{t_{1}}\left(E\left\|\left(S\left(t_{1}+\epsilon-s\right)-S\left(t_{1}-s\right)\right) g(s, x(s, \xi, \lambda), \lambda)\right\|_{L_{2}^{0}}^{p}\right)^{\frac{2}{p}} d s\right)^{\frac{p}{2}} \\
& +2^{p-1} C_{p}\left(\int_{t_{1}}^{t_{1}+\epsilon}\left(E\left\|S\left(t_{1}+\epsilon-s\right) g(s, x(s, \xi, \lambda), \lambda)\right\|_{L_{2}^{0}}^{p}\right)^{\frac{2}{p}} d s\right)^{\frac{p}{2}} \rightarrow 0
\end{aligned}
$$

as $\epsilon \rightarrow 0$, where $C_{p}=(p(2 p-1))^{p}$. Thus

$$
\left\|J_{\lambda}(x)\left(t_{1}+\epsilon\right)-J_{\lambda}(x)\left(t_{1}\right)\right\|_{\chi} \rightarrow 0
$$

as $\epsilon \rightarrow 0$, which implies that $J_{\lambda}$ is continuous in $\chi$ on $[0, T]$. For each $x, y \in \chi$ and $0 \leq t \leq T$, by the Hölder inequality,

$$
E\left\|x^{*} y\right\|_{H} \leq\left(E\|x\|_{H}^{r}\right)^{\frac{1}{r}}\left(E\|y\|_{H}^{s}\right)^{\frac{1}{s}}, \quad x, y \in H, \frac{1}{r}+\frac{1}{s}=1,
$$

where $*$ represents the transpose, and the Lyapunov inequality

$$
\left(E\|x\|_{H}^{m}\right)^{\frac{1}{m}} \leq\left(E\|x\|_{H}^{n}\right)^{\frac{1}{n}}, \quad x \in H, 0<m \leq n<+\infty
$$

and (ii), we have

$$
\begin{aligned}
E \| & (s, x(s, \xi, \lambda), \lambda)-f(s, y(s, \xi, \lambda), \lambda) \|_{H} \\
& \leq c E\left[\|x(s, \xi, \lambda)-y(s, \xi, \lambda)\|_{H}\left(\|x(s, \xi, \lambda)\|_{H}^{q}+\|y(s, \xi, \lambda)\|_{H}^{q}\right)\right] \\
& \leq c\left(E\|x(s, \xi, \lambda)-y(s, \xi, \lambda)\|_{H}^{q+1}\right)^{\frac{1}{q+1}}\left[\left(E\|x(s, \xi, \lambda)\|_{H}^{q+1}\right)^{\frac{q}{q+1}}+\left(E\|y(s, \xi, \lambda)\|_{H}^{q+1}\right)^{\frac{q}{q+1}}\right] \\
& \leq c\left(E\|x(s, \xi, \lambda)-y(s, \xi, \lambda)\|_{H}^{p}\right)^{\frac{1}{p}}\left[\left(E\|x(s, \xi, \lambda)\|_{H}^{p}\right)^{\frac{q}{p}}+\left(E\|y(s, \xi, \lambda)\|_{H}^{p}\right)^{\frac{q}{p}}\right] \\
& \leq 2 c(2 M)^{\frac{q+1}{p}} e^{-\frac{(q+1) \alpha s}{p}} \delta^{\frac{q}{p}}\left(\|x(s, \xi, \lambda)-y(s, \xi, \lambda)\|_{\chi}\right)^{\frac{1}{p}} .
\end{aligned}
$$

By (iii), we obtain

$$
\begin{aligned}
E\|g(s, x(s, \xi, \lambda), \lambda)-g(s, y(s, \xi, \lambda), \lambda)\|_{L_{2}^{0}}^{p} & \leq d^{p} E\|x(s, \xi, \lambda)-y(s, \xi, \lambda)\|_{H}^{p} \\
& \leq 2 M d^{p} e^{-\alpha s}\|x(s, \xi, \lambda)-y(s, \xi, \lambda)\|_{\chi} .
\end{aligned}
$$

Then, by (13), (14) and Lemma 2.1, we have

$$
\begin{aligned}
& E\left\|J_{\lambda}(x)(t, \xi)-J_{\lambda}(y)(t, \xi)\right\|_{H}^{p} \\
& \leq 2^{p-1}\left[E\left\|\int_{0}^{t} S(t-s)(f(s, x(s, \xi, \lambda), \lambda)-f(s, y(s, \xi, \lambda), \lambda)) d s\right\|_{H}^{p}\right. \\
& \left.\quad+E\left\|\int_{0}^{t} S(t-s)(g(s, x(s, \xi, \lambda), \lambda)-g(s, x(s, \xi, \lambda), \lambda)) d \omega(s)\right\|_{H}^{p}\right] \\
& \leq 2^{p-1}\left[\left(\int_{0}^{t} E\|S(t-s)(f(s, x(s, \xi, \lambda), \lambda)-f(s, y(s, \xi, \lambda), \lambda))\|_{H} d s\right)^{p}\right. \\
& \left.\quad+C_{p}\left(\int_{0}^{t}\left(E\|S(t-s)(g(s, x(s, \xi, \lambda), \lambda)-g(s, x(s, \xi, \lambda), \lambda))\|_{L_{2}^{0}}^{p}\right)^{\frac{2}{p}} d s\right)^{\frac{p}{2}}\right]
\end{aligned}
$$




$$
\begin{aligned}
\leq & 2^{p-1}\left[E\left(\int_{0}^{t} M e^{-\eta(t-s)}\|f(s, x(s, \xi, \lambda), \lambda)-f(s, y(s, \xi, \lambda), \lambda)\|_{H} d s\right)^{p}\right. \\
& \left.+C_{p}\left(\int_{0}^{t}\left(M^{p} e^{-p \eta(t-s)} E\|g(s, x(s, \xi, \lambda), \lambda)-g(s, x(s, \xi, \lambda), \lambda)\|_{L_{2}^{0}}^{p}\right)^{\frac{2}{p}} d s\right)^{\frac{p}{2}}\right] \\
\leq & {\left[2^{2 p+q} M^{p+q+1} c^{p} \delta^{q} e^{-\eta p t}\left(\int_{0}^{t} e^{\eta-\frac{(q+1) \alpha s}{p}} d s\right)^{p}\right.} \\
& \left.+2^{p} M^{p+1} d^{p} C_{p} e^{-p \eta t}\left(\int_{0}^{t} e^{\frac{2 p \eta s-2 \alpha s}{p}} d s\right)^{\frac{p}{2}}\right]\|x-y\|_{\chi} \\
\leq & \left\{2^{2 p+q} M^{p+q+1} c^{p} \delta^{q}\left|\frac{p}{p \eta-\alpha(q+1)}\right|^{p}+2^{p} M^{p+1} d^{p} C_{p}\left[\frac{p}{2(p \eta-\alpha)}\right]^{\frac{p}{2}}\right\} \\
& \times e^{-\alpha t}\|x-y\|_{\chi} .
\end{aligned}
$$

Therefore

$$
\left\|J_{\lambda}(x)-J_{\lambda}(y)\right\|_{\chi} \leq \theta\|x-y\|_{\chi}
$$

where $\theta=2^{2 p+q-1} M^{p+q} c^{p} \delta^{q}\left|\frac{p}{p \eta-\alpha(q+1)}\right|^{p}+2^{p-1} M^{p} d^{p} C_{p}\left[\frac{p}{2(p \eta-\alpha)}\right]^{\frac{p}{2}}$. Taking $\delta$ sufficiently small so that $\theta<\frac{1}{2}$, the operator $J_{\lambda}$ becomes a contraction. In addition, we can obtain

$$
\left\|J_{\lambda}(x)\right\| \chi \leq\|S(t) \xi\| \chi+\theta\|x\| \chi \leq \frac{1}{2} \delta+\frac{1}{2} \delta=\delta
$$

This shows that $J_{\lambda}(\chi) \subset \chi$. Thus, there exists a unique function $x \in \chi$ such that $J_{\lambda}(x)=x$. By the above inequality, we have $\|x\|_{\chi} \leq \frac{\|\xi\|_{H}^{p}}{2(1-\theta)}$, which means that (4) holds. Writing $y_{\lambda}=$ $x(\cdot, \xi, \lambda)$, we have

$$
y_{\lambda}-y_{\mu}=J_{\lambda} y_{\lambda}-J_{\mu} y_{\mu}=J_{\lambda} y_{\lambda}-J_{\lambda} y_{\mu}+J_{\lambda} y_{\mu}-J_{\mu} y_{\mu}
$$

for any $\lambda, \mu \in Y$. Thus

$$
\left\|y_{\lambda}-y_{\mu}\right\|_{\chi} \leq \theta\left\|y_{\lambda}-y_{\mu}\right\|_{\chi}+\left\|J_{\lambda} y_{\mu}-J_{\mu} y_{\mu}\right\|_{\chi} .
$$

It follows that

$$
\left\|y_{\lambda}-y_{\mu}\right\|_{\chi} \leq(1-\theta)^{-1}\left\|J_{\lambda} y_{\mu}-J_{\mu} y_{\mu}\right\|_{\chi} .
$$

Using the Lyapunov inequality

$$
\left(E\|x\|_{H}^{m}\right)^{\frac{1}{m}} \leq\left(E\|x\|_{H}^{n}\right)^{\frac{1}{n}}, \quad x \in H, 0<m \leq n<+\infty,
$$

from (ii), (iii) and (4), we get

$$
\begin{aligned}
a(s) & =E\|f(s, x(s, \xi, \mu), \lambda)-f(s, x(s, \xi, \mu), \mu)\|_{H} \\
& \leq c\|\lambda-\mu\|_{Y} E\|x(s, \xi, \mu)\|_{H}^{q+1}
\end{aligned}
$$




$$
\begin{aligned}
& \leq c\|\lambda-\mu\|_{Y}\left(E\|x(s, \xi, \mu)\|_{H}^{p}\right)^{\frac{q+1}{p}} \\
& \leq c(L \delta)^{\frac{q+1}{p}} e^{-\frac{(q+1) s}{p}}\|\lambda-\mu\|_{Y}
\end{aligned}
$$

and

$$
\begin{aligned}
b(s) & =E\|g(s, x(s, \xi, \mu), \lambda)-g(s, x(s, \xi, \mu), \mu)\|_{L_{2}^{0}}^{p} \\
& \leq d^{p}\|\lambda-\mu\|_{Y}^{p} E\|x(s, \xi, \mu)\|_{H}^{p} \\
& \leq d^{p} L \delta e^{-\alpha s}\|\lambda-\mu\|_{Y}^{p} .
\end{aligned}
$$

Thus

$$
\begin{aligned}
\left\|\left(J_{\lambda} y_{\mu}\right)(t)-\left(J_{\mu} y_{\mu}\right)(t)\right\|_{\chi} & \\
= & \frac{1}{2 M} \sup _{t \geq 0}\left[e^{\alpha t} E\left\|\left(J_{\lambda} y_{\mu}\right)(t)-\left(J_{\mu} y_{\mu}\right)(t)\right\|_{H}^{P}\right] \\
\leq & \frac{2^{p-2}}{M} \sup _{t \geq 0}\left\{e ^ { \alpha t } \left[E\left\|\int_{0}^{t} S(t-s)(f(s, x(s, \xi, \mu), \lambda)-f(s, x(s, \xi, \mu), \mu)) d s\right\|_{H}^{p}\right.\right. \\
& \left.\left.+E\left\|\int_{0}^{t} S(t-s) g(s, x(s, \xi, \mu), \lambda)-g(s, x(s, \xi, \mu), \mu) d \omega(s)\right\|_{H}^{p}\right]\right\} \\
\leq & \frac{2^{p-2}}{M} \sup _{t \geq 0}\left\{e ^ { \alpha t } \left[\left(\int_{0}^{t} E\|S(t-s)(f(s, x(s, \xi, \mu), \lambda)-f(s, x(s, \xi, \mu), \mu))\|_{H} d s\right)^{p}\right.\right. \\
& \left.\left.+C_{p}\left(\int_{0}^{t}\left(E\|S(t-s)(g(s, x(s, \xi, \mu), \lambda)-g(s, x(s, \xi, \mu), \mu))\|_{L_{2}^{0}}^{p}\right)^{\frac{2}{p}} d s\right)^{\frac{p}{2}}\right]\right\} \\
\leq & 2^{p-2} M^{p-1} \sup _{t \geq 0}\left\{e^{\alpha t}\left[\left(\int_{0}^{t} e^{-\eta(t-s)} a(s) d s\right)^{p}+C_{p}\left(\int_{0}^{t}\left(e^{-p \eta(t-s)} b(s)\right)^{\frac{2}{p}} d s\right)^{\frac{p}{2}}\right]\right\} \\
\leq & 2^{p-2} M^{p-1} \sup _{t \geq 0}\left\{e ^ { \alpha t } \left[c^{p}(L \delta)^{q+1}\left(\int_{0}^{t} e^{\frac{-p \eta t+p \eta s-(q+1) \alpha s}{p}} d s\right)^{p}\right.\right. \\
& \left.\left.+L \delta d^{p} C_{p}\left(\int_{0}^{t}\left(e^{-p \eta(t-s)-\alpha s}\right)^{\frac{2}{p}} d s\right)^{\frac{p}{2}}\right]\right\}\|\lambda-\mu\|_{Y}^{p} \\
\leq & \theta_{1}\|\lambda-\mu\|_{Y}^{p},
\end{aligned}
$$

where $\theta_{1}=2^{p-2} M^{p-1} c^{p}(L \delta)^{q+1}\left|\frac{p}{p \eta-\alpha(q+1)}\right|^{p}+2^{p-2} M^{p-1} d^{p} C_{p} L \delta\left[\frac{p}{2(p \eta-\alpha)}\right]^{\frac{p}{2}}$. Therefore

$$
\left\|y_{\lambda}-y_{\mu}\right\|_{\chi} \leq \frac{\theta_{1}}{1-\theta}\|\lambda-\mu\|_{Y}^{p}
$$

which yields (5). This completes the proof of the theorem.

Remark 3.1 From the proof of Theorem 3.1, if we take $\delta$ sufficiently small so that $\theta<\frac{1}{2}$, the mild solutions (1) with $\delta$-domain initial value are $p$ moment locally exponentially stable. In this case, the property of $p$ moment stability is local. 
Theorem 3.2 Assume that (i)-(v) hold. Then, for any $\delta>0$ sufficiently small, mild solution $x(t, \xi, \lambda)$ of $(1)$ is almost surely exponentially stable, that is, there exists $0<\alpha<p \eta$ such that

$$
\limsup _{t \rightarrow+\infty} \frac{1}{t} \log \|x(t, \xi, \lambda)\|_{H} \leq-\frac{\alpha}{2 p}, \quad \text { a.s. }
$$

for any $\lambda \in Y$ and $\xi \in B(\delta)$. Furthermore,

$$
\limsup _{t \rightarrow+\infty} \frac{1}{t} \log \frac{\|x(t, \xi, \lambda)-x(t, \xi, \mu)\|_{H}}{\|\lambda-\mu\|_{Y}} \leq-\frac{\alpha}{2 p} \text {, a.s. }
$$

for any $\lambda, \mu \in Y, \lambda \neq \mu$ and $\xi \in B(\delta)$.

Proof For any fixed positive real number $\varepsilon_{N}>0(N=2,3, \ldots)$,

$$
\begin{aligned}
& P\left(\sup _{N \leq t \leq N+1}\|x(t, \xi, \lambda)\|_{H}>\varepsilon_{N}\right) \\
& \leq\left(\frac{3}{\varepsilon_{N}}\right)^{p} E\left(\sup _{N \leq t \leq N+1}\|S(t-N) x(N, \xi, \lambda)\|_{H}^{p}\right) \\
&+\left(\frac{3}{\varepsilon_{N}}\right)^{p} E\left(\sup _{N \leq t \leq N+1}\left\|\int_{N}^{t} S(t-s) f(s, x(s, \xi, \lambda), \lambda) d s\right\|^{p}\right) \\
&+\left(\frac{3}{\varepsilon_{N}}\right)^{p} E\left(\sup _{N \leq t \leq N+1}\left\|\int_{N}^{t} S(t-s) g(s, x(s, \xi, \lambda), \lambda) d \omega(s)\right\|_{H}^{p}\right) \\
&= \sum_{i=1}^{3} I_{i} .
\end{aligned}
$$

By (i) and (4), we have

$$
I_{1} \leq\left(\frac{3}{\varepsilon_{N}}\right)^{p} M^{p} E\|x(N, \xi, \lambda)\|_{H}^{p} \leq\left(\frac{3}{\varepsilon_{N}}\right)^{p} M^{p} L \delta e^{-\alpha N} .
$$

It follows from (ii) and (4) that

$$
\begin{aligned}
E \| f\left(s, x(s, \xi, \lambda), \lambda \|_{H}\right. & \leq c E\|x(s, \xi, \lambda)\|^{q+1} \\
& \leq c\left(E\|x(s, \xi, \lambda)\|^{p}\right)^{\frac{q+1}{p}} \leq c(L \delta)^{\frac{q+1}{p}} e^{-\frac{(q+1) \alpha s}{p}} .
\end{aligned}
$$

This implies that

$$
\begin{aligned}
I_{2} & \leq c^{p} M^{p}(L \delta)^{q+1}\left(\frac{3}{\varepsilon_{N}}\right)^{p} \sup _{N \leq t \leq N+1}\left\|\int_{N}^{t} e^{-\eta(t-s)} e^{-\frac{(q+1) \alpha s}{p}} d s\right\|^{p} \\
& \leq c^{p} M^{p}(L \delta)^{q+1}\left(\frac{3}{\varepsilon_{N}}\right)^{p}\left|\frac{p}{p \eta-(q+1) \alpha}\right|^{p} e^{-\alpha N} .
\end{aligned}
$$

From (iii) and (4), we have the following estimation:

$$
E \| g\left(s, x(s, \xi, \lambda), \lambda\left\|_{L_{2}^{0}}^{p} \leq d^{p} E\right\| x(s, \xi, \lambda) \|_{H}^{p} \leq d^{p} L \delta e^{-\alpha s},\right.
$$


and

$$
\begin{aligned}
I_{3} & \leq M^{p} C_{p}\left(\frac{3}{\varepsilon_{N}}\right)^{p} \sup _{N \leq t \leq N+1}\left(\int_{N}^{t}\left(e^{-p \eta(t-s)} E\|g(s, x(s, \xi, \lambda), \lambda)\|_{L_{2}^{0}}^{p}\right)^{\frac{2}{p}} d s\right)^{\frac{p}{2}} \\
& \leq M^{p} d^{p} C_{p} L \delta\left(\frac{3}{\varepsilon_{N}}\right)^{p} \sup _{N \leq t \leq N+1}\left(\int_{N}^{t}\left(e^{-p \eta(t-s)} e^{-\alpha s}\right)^{\frac{2}{p}} d s\right)^{\frac{p}{2}} \\
& \leq M^{p} d^{p} C_{p} L \delta\left(\frac{3}{\varepsilon_{N}}\right)^{p}\left[\frac{p}{2(p \eta-\alpha)}\right]^{\frac{p}{2}} e^{-\alpha N} .
\end{aligned}
$$

Substituting (28), (30) and (32) into (27), we have

$$
P\left\{\sup _{N \leq t \leq N+1}\|x(t, \xi, \lambda)\|_{H}>\varepsilon_{N}\right\} \leq \kappa\left(\frac{3}{\varepsilon_{N}}\right)^{p} e^{-\alpha N}
$$

where $\kappa=M^{p} L \delta+c^{p} M^{p}(L \delta)^{q+1}\left|\frac{p}{p \eta-(q+1) \alpha}\right|^{p}+M^{p} d^{p} C_{p} L \delta\left[\frac{p}{2(p \eta-\alpha)}\right]^{\frac{p}{2}}$. As $\varepsilon_{N}$ is an arbitrarily given real number, let $\varepsilon_{N}=e^{-\frac{\alpha N}{2 p}}$ such that

$$
P\left\{\sup _{N \leq t \leq N+1}\|x(t, \xi, \lambda)\|_{H}>e^{-\frac{\alpha N}{2 p}}\right\} \leq \kappa 3^{p} e^{-\frac{\alpha N}{2}}
$$

Consequently, from the Borel-Cantelli lemma, there exists $\bar{T}(\omega)>0$ such that for any $t \geq$ $\bar{T}(\omega)$,

$$
\|x(t, \xi, \lambda)\|_{H}^{p} \leq e^{-\frac{\alpha t}{2 p}}, \quad \text { a.s. }
$$

It remains to verify that (26) holds. The argument is similar to the above proof of (27). For any $\varepsilon_{N}>0(N=2,3, \ldots)$,

$$
\begin{aligned}
P\left(\sup _{N \leq t \leq N+1} \frac{\|x(t, \xi, \lambda)-x(t, \xi, \mu)\|_{H}}{\|\lambda-\mu\|_{Y}}>\varepsilon_{N}\right) & \\
\leq & \left(\frac{3}{\varepsilon_{N}}\right)^{p} E\left(\sup _{N \leq t \leq N+1} \frac{\|S(t-N)(x(N, \xi, \lambda)-x(N, \xi, \mu))\|_{H}^{p}}{\|\lambda-\mu\|_{Y}^{p}}\right) \\
& +\left(\frac{3}{\varepsilon_{N}}\right)^{p} E\left(\sup _{N \leq t \leq N+1} \frac{\left\|\int_{N}^{t} S(t-s)(f(s, x(s, \xi, \lambda), \lambda)-f(s, x(s, \xi, \mu), \mu)) d s\right\|^{p}}{\|\lambda-\mu\|_{Y}^{p}}\right) \\
& +\left(\frac{3}{\varepsilon_{N}}\right)^{p} E\left(\sup _{N \leq t \leq N+1} \frac{\left\|\int_{N}^{t} S(t-s)(g(s, x(s, \xi, \lambda), \lambda)-g(s, x(s, \xi, \mu), \mu)) d \omega(s)\right\|_{H}^{p}}{\|\lambda-\mu\|_{Y}^{p}}\right) \\
= & \sum_{i=1}^{3} I_{i}^{\prime} .
\end{aligned}
$$

From (i) and (5), we obtain

$$
I_{1}^{\prime} \leq\left(\frac{3}{\varepsilon_{N}}\right)^{p} M^{p} K e^{-\alpha N}
$$


By (4), (5) and (ii), applying inequalities (12) and (13), we get

$$
\begin{aligned}
& E\|f(s, x(s, \xi, \lambda), \lambda)-f(s, x(s, \xi, \mu), \mu)\|_{H} \\
& \leq E\|f(s, x(s, \xi, \lambda), \lambda)-f(s, x(s, \xi, \mu), \lambda)\|_{H} \\
&+E\|f(s, x(s, \xi, \mu), \lambda)-f(s, x(s, \xi, \mu), \mu)\|_{H} \\
& \leq c E\left[\|x(s, \xi, \lambda)-x(s, \xi, \mu)\|_{H}\left(\|x(s, \xi, \lambda)\|^{q}+\|x(s, \xi, \mu)\|^{q}\right)\right] \\
&+c E\|\lambda-\mu\|_{Y}\|x(s, \xi, \mu)\|_{H}^{q+1} \\
& \leq c\left(E\|x(s, \xi, \lambda)-x(s, \xi, \mu)\|_{H}^{p}\right)^{\frac{1}{p}}\left[\left(E\|x(s, \xi, \lambda)\|^{p}\right)^{\frac{q}{p}}+\left(E\|x(s, \xi, \mu)\|^{p}\right)^{\frac{q}{p}}\right] \\
&+c\|\lambda-\mu\|_{Y} E\left(\|x(s, \xi, \mu)\|_{H}^{p}\right)^{\frac{q+1}{p}} \\
& \leq c\left[2 K^{\frac{1}{p}}(L \delta)^{\frac{q}{p}}+(L \delta)^{\frac{q+1}{p}}\right] e^{-\frac{(q+1) \alpha s}{p}}\|\lambda-\mu\|_{Y} .
\end{aligned}
$$

This implies that

$$
\begin{aligned}
I_{2}^{\prime} & \leq c^{p} M^{p}\left(\frac{3}{\varepsilon_{N}}\right)^{p}\left[2 K^{\frac{1}{p}}(L \delta)^{\frac{q}{p}}+(L \delta)^{\frac{q+1}{p}}\right]^{p} \sup _{N \leq t \leq N+1}\left\|\int_{N}^{t} e^{-\eta(t-s)} e^{-\frac{(q+1) \alpha s}{p}} d s\right\|^{p} \\
& \leq c^{p} M^{p}\left(\frac{3}{\varepsilon_{N}}\right)^{p}\left[2 K^{\frac{1}{p}}(L \delta)^{\frac{q}{p}}+(L \delta)^{\frac{q+1}{p}}\right]^{p}\left|\frac{p}{p \eta-(q+1) \alpha}\right|^{p} e^{-\alpha N} .
\end{aligned}
$$

It follows from (4), (5) and (iii) that

$$
\begin{aligned}
E \| & g(s, x(s, \xi, \lambda), \lambda)-g(s, x(s, \xi, \mu), \mu) \|_{L_{2}^{0}}^{P} \\
\leq & 2^{P-1}\left[E\|g(s, x(s, \xi, \lambda), \lambda)-g(s, x(s, \xi, \mu), \lambda)\|_{L_{2}^{0}}^{P}\right. \\
& \left.+E\|g(s, x(s, \xi, \mu), \lambda)-g(s, x(s, \xi, \mu), \mu)\|_{L_{2}^{0}}^{P}\right] \\
& \leq 2^{p-1} d^{p}\left[E\|x(s, \xi, \lambda)-x(s, \xi, \mu)\|_{H}^{P}+\|\lambda-\mu\|_{Y}^{p} E\|x(s, \xi, \mu)\|_{H}^{P}\right] \\
\leq & 2^{p-1} d^{p} \delta(L+K) e^{-\alpha s}\|\lambda-\mu\|_{Y}^{p} .
\end{aligned}
$$

So

$$
\begin{aligned}
I_{3}^{\prime} & \leq 2^{p-1} d^{p} M^{p} \delta C_{p}(L+K)\left(\frac{3}{\varepsilon_{N}}\right)^{p} \sup _{N \leq t \leq N+1}\left(\int_{N}^{t}\left(e^{-p \eta(t-s)} e^{-\alpha s}\right)^{\frac{2}{p}} d s\right)^{\frac{p}{2}} \\
& \leq 2^{p-1} d^{p} M^{p} \delta C_{p}\left(\frac{3}{\varepsilon_{N}}\right)^{p}(L+K)\left[\frac{p}{2(p \eta-\alpha)}\right]^{\frac{p}{2}} e^{-\alpha N} .
\end{aligned}
$$

Substituting (37), (39) and (41) into (36), we have

$$
P\left(\sup _{N \leq t \leq N+1} \frac{\|x(t, \xi, \lambda)-x(t, \xi, \mu)\|_{H}}{\|\lambda-\mu\|_{Y}}>\varepsilon_{N}\right) \leq \bar{\kappa}\left(\frac{3}{\varepsilon_{N}}\right)^{p} e^{-\alpha N}
$$

where $\bar{\kappa}=M^{p} K \delta+c^{p} M^{p}\left(\frac{3}{\varepsilon_{N}}\right)^{p}\left[2 K^{\frac{1}{p}}(L \delta)^{\frac{q}{p}}+(L \delta)^{\frac{q+1}{p}}\right]^{p}\left|\frac{p}{p \eta-(q+1) \alpha}\right|^{p}+2^{p-1} d^{p} M^{p} \delta C_{p}(L+K) \times$ $\left[\frac{p}{2(p \eta-\alpha)}\right]^{\frac{p}{2}}$. We can proceed to the remaining proof in a similar manner. 


\section{Example}

In this section, an example is provided to illustrate our results.

Example 4.1 Consider the following stochastic partial equation:

$$
\begin{aligned}
& d Z(t, x)=\left[\frac{\partial^{2}}{\partial x^{2}} Z(t, x)+\frac{\lambda^{2} Z^{2}(t, x)}{1+|Z(t, x)|}\right] d t+\frac{\lambda Z(t, x)}{20(1+|Z(t, x)|)} d \omega(t), \quad t \geq 0, \\
& Z(t, 0)=Z(t, \pi)=0, \quad t \geq 0, \\
& Z(0, x)=\xi(x),
\end{aligned}
$$

where $\omega(t)$ is a standard one-dimensional Brownian motion, $\lambda \in Y=\left[\frac{1}{4}, \frac{1}{3}\right]$ is a parameter. Let $A=\frac{\partial^{2}}{\partial x^{2}}$ with the domain

$$
\mathcal{D}(A)=\left\{u \in H: \frac{\partial}{\partial u}, \frac{\partial^{2}}{\partial u^{2}} \in H, u(0)=u(\pi)=0\right\},
$$

then $\|S(t)\|_{H} \leq e^{-t}, \forall t \geq 0$, which implies that $M=1$. It is easy to see that (ii)-(iii) hold and $c=1, q=1, d=\frac{1}{20}$. Taking $p=4$, we may show that $2^{p} d^{p} C_{p}\left(\frac{1}{2 \pi^{2}}\right)^{\frac{p}{2}}<1$. Then the mild solution of Eq. (43) has locally stable behavior and parameter dependence for $\delta>0$ sufficiently small.

\section{Conclusion}

This paper is devoted to locally stable behavior and parameter dependence for stochastic differential equations. Our results are derived under sufficiently small Lipschitz perturbation in the parameter system. Meanwhile, a lot of stochastic inequality techniques are used to estimate the bound of mild solution. The future research topics would be extending locally stable behavior and parameter dependence to the stochastic delayed parameter system.

\section{Competing interests}

The author declares that they have no competing interests.

\section{Author's contributions}

The author contributed to the manuscript. The author read and approved the final manuscript.

\section{Acknowledgements}

The author would like to thank the referee and the associate editor for their very helpful suggestions.

Received: 19 May 2014 Accepted: 8 October 2014 Published: 27 Oct 2014

\section{References}

1. Karatzas, I, Shreve, SE: Brownian Motion and Stochastic Calculus. Springer, Berlin (1991)

2. Da Prato, G, Zabczyk, J: Stochastic Equations in Infinite Dimensions. Cambridge University Press, Cambridge (1992)

3. Kan, X, Shu, H, Li, Z: Almost sure state estimation for nonlinear stochastic systems with Markovian switching. Nonlinear Dyn. 76, 1591-1602 (2014)

4. Xiong, P, Huang, L: On pth moment exponential stability of stochastic fuzzy cellular neural networks with time-varying delays and impulses. Adv. Differ. Equ. 2013, 172 (2013)

5. Hu, J, Wang, Z, Shen, B, Gao, H: Quantised recursive filtering for a class of nonlinear systems with multiplicative noises and missing measurements. Int. J. Control 86, 650-663 (2013)

6. Haussmann, UG: Asymptotic stability of the linear Itô equation in infinite dimensions. J. Math. Anal. Appl. 65, 219-235 (1978)

7. Caraballo, T: Asymptotic exponential stability of stochastic partial differential equations with delay. Stochastics 33 , 27-47 (1990)

8. Mao, X: Stochastic Differential Equations and Applications. Ellis Horwood, Chichester (1997) 
9. Caraballo, T, Real, J: Partial differential equations with delayed random perturbations: existence, uniqueness, and stability of solutions. Stoch. Anal. Appl. 11,497-511 (1993)

10. Govindan, TE: Stability of mild solutions of stochastic evolution equations with variable delay. Stoch. Anal. Appl. 21, 1059-1077 (2003)

11. Govindan, TE: Existence and stability of solutions of stochastic semilinear functional differential equations. Stoch. Anal. Appl. 20, 1257-1280 (2002)

12. Caraballo, T, Liu, K: Exponential stability of mild solutions of stochastic partial differential equations with delays. Stoch. Anal. Appl. 17, 743-763 (1999)

13. Liu, K, Mao, X: Exponential stability of non-linear stochastic evolution equations. Stoch. Process. Appl. 78, 173-193 (1998)

14. Da Prato, G, Iannelli, M, Tubaro, L: Semilinear stochastic differential equations in Hilbert spaces. Boll. Unione Mat. Ital., A 16, 168-185 (1979)

15. Dawson, DA: Stochastic evolution equations and related measured processes. J. Multivar. Anal. 5, 1-52 (1975)

16. Pazy, A: Semigroups of Linear Operators and Applications to Partial Differential Equations. Springer, New York (1983)

17. Taniguchi, T, Liu, K, Truman, A: Existence, uniqueness, and asymptotic behavior of mild solutions to stochastic functional differential equations in Hilbert spaces. J. Differ. Equ. 181, 72-91 (2002)

18. Burton, TA: Stability by Fixed Point Theory for Functional Differential Equations. Dover, New York (2006)

19. Luo, J: Fixed points and exponential stability of mild solutions of stochastic partial differential equations with delays. J. Math. Anal. Appl. 342, 753-760 (2008)

20. Sakthivel, R, Luo, J: Asymptotic stability of impulsive stochastic partial differential equations with infinite delays. J. Math. Anal. Appl. 356, 1-6 (2009)

10.1186/1687-1847-2014-276

Cite this article as: Pan: Local stability and parameter dependence of mild solutions for stochastic differential equations. Advances in Difference Equations 2014, 2014:276

\section{Submit your manuscript to a SpringerOpen ${ }^{\circ}$ journal and benefit from:}

- Convenient online submission

- Rigorous peer review

- Immediate publication on acceptance

Open access: articles freely available online

High visibility within the field

- Retaining the copyright to your article 